\title{
Función muscular esquelética en pacientes que han sufrido desnutrición
}

\author{
Jorge Alvear A. ${ }^{1}$; Samuel Ruiz N. ${ }^{2}$ \\ Muscle contraction in children after recovery from \\ severe infantile protein calorie malnutrition
}

\begin{abstract}
Ske etal muscle function was compared in forly ciden aged ' $2 \pm 4 \mathrm{~m}$ who hod been recovered from protein cosie malnutrition early in life [study groupl and a smilar number of control subjects paired by age cnd sex without antecederit nutritional disorder. Skeletol muscle function was evaluated by measuring ine force of isometric contraction of the adductor pollicis muscle of the thumo after electric simutation of the unlar neve at line wrist by means of a st:rulus isolation unit (Grass Model FT-10) fellowing the Shizgal orolocol. Muscle function was relaled ro bady composition obtained by Frisancho and Durnin formula. The forces genealed during stimuation cnd recovery periods were both reduced $f p<0,05 t$ in the study group when compared to controls. Early protern calo:e malnutrition modifies skelelal muscle function in children several vears after reccuery
\end{abstract}

(Key words: riutritional discrders, molnutsition, muscte controction.]

El músculo es la reserva más importante de proteina disponible del cuerpo, la que se ve reducida considerablemente por la desnutrición grave en lumanos y en animales de experimentación'. Durante el ayuno parcial o total, la proteína corporal es destruida para proporcionar aminoácidos, lo que se traduce en pérdida de masa corporal total. Esta última representa e] número total de células funcionales metabolicamente activas, por lo tanto, la fuente de consumo de $\mathrm{O}_{2}$ y de producción de $\mathrm{CO}_{2}{ }^{2}$. La masa muscular en niños desnutridos puede estar disminuida hasta en $70 \%^{3}$. Las biopsias musculares muestran en ellos, al compararlos con niños que se han recuperado de desnutrición, reducciones de hasta $45 \%$ de la proteína total, las que comprometen el número y el tamaño de las fibras musculares ${ }^{4.5}$. Durante la recuperación de la desnutrición ocurre un período đe ganancia

1. Unidad de Nutrición Clínica, Instituto de Nutrición y Tecnología de los Alimentos (INTA), Universidad de Chile.

2. Unidad de Neurofisiologia y Biofísica, Instituto de Nutrición y Tecnología de los Alimentos (INTA), Unvversidad de Chile.

Financiado por DT] M2400 - 8722. rápida de peso en concordancia con aumento de la excreción de creatinina, por lo que ésta ha sido usada como método indirecto para indicar incrementos de masa muscular ${ }^{6}$. Además, durante la recuperación nutricional aumenta la masa muscular en proporción mayor que la de aumento del peso corporal, que puede diferir según el grado inicial de retraso en talla o la pérdida de peso ${ }^{3.8}$. Estas observaciones indican que la masa muscular es muy influida por el estado nutricional, sufriendo cambios adaptativos importantes ante la injuria nutricional y en su rehabilitación.

La función de los músculos puede ser evaluada por su capacidad de trabajo. La escala MRC (Medical Research Council) es un sistema para estimar potencia muscular (capacidad de trabajo por unidad de (iempo), que considera cinco grados de eficiencia, desde ausencia de contracción a contracción normal. Ciertos músculos no son afectados por la fuerza de gravedad, como los encargados de la rotación de dedos de pies y manos, lo que permite medir en ellos la fuerza de su contracción sin distorsiones significativas atribuibles a dicha fuerzat to.

Por otra parte, el músculo aductor del pulgar pertenece al grupo de músculos de contracción 
rápida" que es afectado por el ayuno aumentando la actividad glicolítica, por lo que resulta susceptible a la fatiga. Para producir contracción muscular, la estimulación eléctrica tiene las ventajas de subsanar problemas como la falta de motivación $y$, si bien no es un cstímulo "fisiológico", contar con la cualidad de ser controlable.

La influencia del estado nutricional sobre Ia función muscular ha sido poco estudiada. El proposito de este trabajo fue determinar si la desnutrición grave precoz tiene efectos sobre la función muscular esquelética, varios años después de la recuperación nutricional.

\section{Material y Método}

Se estudiaron niños sometidos a un seguimiento iongitudinal de 15 años, realizado por el Instituto de Nutrición y Tecnología de los Alimentos de la Universidad de Chile (INTA), cuyas caraclerísticas han sido descritas anteriormente ${ }^{12}, 1.5$. Se incluyo un grupo de estudio, formado por pacientes que habian sufrido desnutricion calórica proteica grave antes de los dos años de vida (20\} niñas y 20 varones) - de la cual se habian recuperado satisfactoriamnente- y uno de control sin este antecedente, con igual número de participantes. Todos los participantes fueron informados de la experiencia que se realizaria y firmaron su consentimiento de acuerdo a las normas étıcas aceptadas para investigación en seres humanos. En ellos se midio peso (con ropa interior, sin zapalos, a la misna hora de la mañana en una balanza Neta), talla de pie, talla sentada (en antropómetro fijo a la pared y silla de medición adaptada para este menester), circunferencias de cráneo y brazo (usando huincha de fibra de vidrio inextensible), pliegues cutáneos (mediante calibrador de Lange). Todas las medi. cjones se reslizaron en el hemicuerpo izquierdols. Se evalus además su composición corporal midiendo área magra y grasa braquial (formola de Frisancho) y el parcentaje de grasa corporal (fónnula de Dumin) ${ }^{\text {ls. } 16}$. El estado nutricional se evaluó por medio del indice peso para taIla $(\mathrm{P} / \mathrm{T})$, la estacura por la relación talla para edad (T/E) adecuándolas al percentil 50 de la tablas ONS ${ }^{17}$. Ademis se hicieron mediciones de hemogiobina y ferritina sérica, corno otro indicador del estado nutricional ${ }^{18}$

La función muscular esquelética fue evaluada con el método Jescrito por Merton y modificado por Edwards'9, que mide la fuerza de la contraccion isometrica ejercida por el dedo pulgar de la mano izquierda. Las mediciones se realizaron después de un buen desayuno. Se ubicó el antebrazo y el brazo en un instrumento que pemite asegurar la inmovilidad relativa del brazo y mano en posición supitia, de tal manera de dejar los dedos semjflectados, con excepción del dedo pulgar, el que se dejó en exiensión. Con el dedo pulgar en abducctón máxina, se ubicó un lazo en la unión interfalángica, el que se conectó a un transductor de ruerza, que mide In tensión efectuada. En todos los experimentos se hicieron al menos dos mediciones: contracción voluntaria (en la que se solicitó al sujeto tensar el dedo al recibir una orden), y por estimulación eléctrica tnediante un par de electrodos de superficie sobte el nervio cubital, para producir una contracción isométrica del músculo aductor del pulgar. En todas las mediciones cada sujeto efectuó 10 elonguciones tratando de unifornar el procedimiento en ambos grupos de estudio.

Cada estímulo produce una contracción del músculo aductor del pulgar qae fue registrada con un transductor de fuerza (Grass Mod FT-10) previamente calibrado. Las scñales fueron amplificadas en un preamplificador Grass Modelo RPS-107, y desplegadas en un osciloscopio Tektronik Mod 2213, cuya interfase de regisiro y vigilancia era un microcomputador Rockwell AIM-60 análogo digital. Las señales fueron registradas en una grabadora magnética para su análisis posterior, en que se midió la amplicud vértice a vértice de tada una de las contracciones voluntarias en las respuestas obtenidas por estitmulación eléctrica, el tamajoo de ellas vértice a vértice con diferentes frecuencias y voliajes de estimulación.

El esiimulador fue disefiado y construido en nuestro Iaboratorio y permite variar la frecuencia $(\mathrm{Hz})$ y la diferencia de potencial del estrmulo. El generador de putso produce senales rectangulares de amplitud variable a voluntad, de 60 a 120 volts y duración constante de $0,2 \mathrm{~ms}$ de duración. Cada registro tenía una duración $2000 \mathrm{~ms}$, lo que permnitia observar las variaciones de amplitud mientras se registraba. Se elaboró una curva de fuerza para frecuencia, estimulando el nervio cubital a voltajes supramáximas con pulsos de frecuencias de $0.5,10,25,50$ y $100 \mathrm{~Hz}$, de ondas unidireccionales de 0,2 ms de duracion. Para contracciones sobre $0,5 \mathrm{~Hz}$ los impulsos dumban I s.

Para compensar las diferencias individuales de musculatura enire los sujetos, la respuesta a la estimulación electrica se expreso como por ciento del máximo de fuerza generado. La fatiga muscular se estudió con el protocolo de Shizgal 2, estimulando el ncrvio cubital con impulsos repetidos de $20 \mathrm{~Hz}$ y de 0,2 ms de duración por $330 \mathrm{~ms}$. La fatigabilidad fue evaluada cada segundo por $120 \mathrm{~s}$, determinando la fuerza de contracción isomeurica a intervalos de 30 s (período de fatiga). La velocidad de recuperación de la fatiga nuscular fue delerminada inmediatamente después del periodo de fatiga, con estímulos de $330 \mathrm{~ms}$ a 20 Hz y $0.2 \mathrm{~ms}$ de duración. Los resultados fueron evaluados y promediados para cada grupo. considerando los promedios de contracciones voluntarias y los de contracciones por estimulación eléctrica del nervio cubital. El análisis estadístico de los resultados fue hecho empleando análisis de varianza (prueba F), prueba $t$ de Student, chi cuadrado. Los promedios de edad se obtuvieron con el método de probito.

Este estudio fue aprobado por el Comité de Etica del INTA, Úniversidad de Chile.

\section{Resultados}

Las medidas antropométricas mostraron diferencias significativas ( $p<0,001$ ) de $8 \mathrm{~kg}$ en el peso, $8 \mathrm{~cm}$ en la talla de pie, $3 \mathrm{~cm}$ en el segmento superior (tajla sentada) y $2 \mathrm{~cm}$ en circunferencia de cráneo entre los varones y de $11 \mathrm{~kg}$ en 
el peso, $11 \mathrm{~cm}$ en la talla y de $6 \mathrm{~cm}$ en el segmento superior y $1,8 \mathrm{~cm}$ en la circunferencia de crâneo en las niñas, en favor de los controles (tabla).

Las curvas de fuerza para la frecuencia del estímulo $(\mathrm{kg} / \mathrm{Hz})$ fueron similares en los dos grupos estudiados independientes del sexo. No hubo diferencia significativa en la fatigabilidad muscular, sitn embargo la recuperación a la fatiga muscular en los niños sin antecedentes de desnutrición fue significativamente más rápida. alcanzando a $90 \%$, a diferencia del grupo experimental que sólo llegaba a $85 \%$ de respuestas frente al estímulo ( $p<0.05$ ) (fïgura 1 ).

Las respuestas a la estimulación eléctrica de los niños controles y niños recuperados fueron diferentes a 25 y $50 \mathrm{~Hz}(\mathrm{p}<0.05)$ (figura 2$)$. Cuando la respuesta a la estimulación muscular se expresó como fuerza absoluta desarrollada y no como porcentaje del máximo, aparecieron diferencias significativas en fatigabilidad y recuperación a la fatiga, siendo mayor la fuerza generada en el grupo control (figura 3). En las curvas no se incluyen las desviaciones estándar para facilitar la expresión gráfica.

No se encontró correlación entre composición corporal y función muscular esquelética (área magra obtenida según Frisancho), con fuerza generada expresada en $\mathrm{kg}$ con una estimulación eléctrica nerviosa a frecuencia de 100 $\mathrm{Hz}$, al final del período de fatiga $100 \mathrm{~s}$ y al

Tabla

Antropometría de los niños esludiados

\begin{tabular}{|c|c|c|c|c|c|c|}
\hline & \multicolumn{3}{|c|}{ Contrales } & \multicolumn{3}{|c|}{ Estudio } \\
\hline & Ho & bres & Mujeres & Ho & bres & Mujeres \\
\hline \multirow{3}{*}{ Peso } & $\mathrm{x}$ & 40,9 & 41.5 & $x$ & 32,8 & 30,3 \\
\hline & $\mathrm{DE}$ & 5,9 & $6,8^{*}$ & $\mathrm{DE}$ & 7,5 & 8.4 \\
\hline & n & 20 & 20 & $n$ & 20 & 20 \\
\hline \multirow{3}{*}{ Talla } & $\mathrm{x}$ & 146.4 & 146,5 & $x$ & $13 B, 3$ & 135,2 \\
\hline & DE & 7,4 & $7.1 *$ & DE & 10.1 & 9.2 \\
\hline & $\mathrm{r}$ & 20 & 20 & ta & 20 & 20 \\
\hline \multirow{3}{*}{ SS } & $x$ & 76,1 & 77,4 & $\mathrm{x}$ & 72.7 & 70,8 \\
\hline & $\mathrm{DE}$ & 4,3 & $5,2 *$ & $\mathrm{DE}$ & 5,4 & 6,3 \\
\hline & $n$ & 20 & 20 & $\mathrm{n}$ & 20 & 20 \\
\hline \multirow{3}{*}{$\mathrm{CC}$} & $\mathrm{x}$ & 54,0 & 53.8 & $\mathrm{x}$ & 52,3 & 52,0 \\
\hline & $\mathrm{DE}$ & 2,3 & $2,5^{*}$ & $\mathrm{DE}$ & 3,4 & 3,2 \\
\hline & $\pi$ & 20 & 20 & $\pi$ & 20 & 20 \\
\hline
\end{tabular}

$* p<0,001$

SS : segmento superior: CC: circunferencia de cráneo

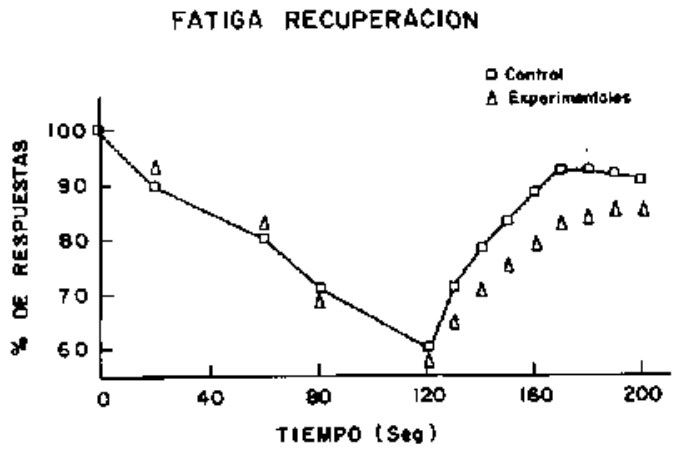

Figura 1: Respuesta del músculo abductor del puigar, como porcentaje de la respuesta máxima, posestimulación de nervio cubital a voltajes de 0.5 a $100 \mathrm{~Hz}$, en niños controles y estudio

FUERZA FRECUENCIA



Figura 2: Respuesta muscular como porcentaje de respuestas a estimulacion nerviosa una vez cada segundo por $120 \mathrm{seg}$ (período de fariga) seguido a estimulación cada $10 \mathrm{seg}$ por $100 \mathrm{seg}$ (recuperación) en niños controles y estudio.

final del período de recuperación $220 \mathrm{~s}$. No se observaron diferencias significativas del estado nutricional de hierro entre los grupos estudiados.

\section{Comentario}

Es corriente observar que la desnutrición calórico-proteica se acompaña de pérdida de masa y debilidad muscular. La disminución de masa muscular se debe en estos casos principalmente a reducción del tamaño de las fibras más que a su número. Existen en la literatura antecedentes que relacionan al estado nutricional y la fuerza muscular medida con dinamómetro ${ }^{20}$, con resul- 


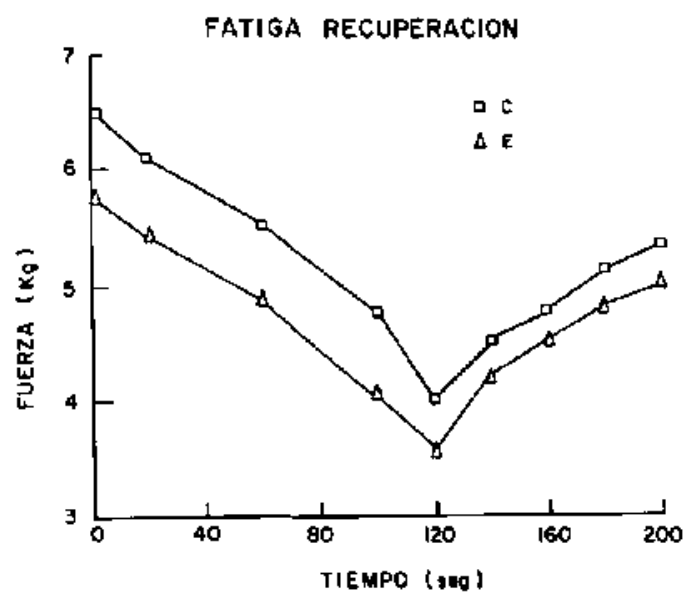

Figura 3: Fuerza generada en kilogramos posestimulación de] nervio cubital, a ftecuencias de una vez cada segundo por $120 \mathrm{seg}$ (Íatiga) seguido par estimulación cada $10 \mathrm{seg}$ por 100 seg (recuperación) en niños controles (promedios y $\mathrm{DE}$ ).

tados muy variables en las mediciones, Io que sugiere que el dinamómetro de mano, adecuado en terreno para estimar estado nutricional de las poblaciones, es poco apropiado para evaluar el de un sujeto, aun cuando resulta útil como indicador de debilidad muscular (para fisioterapeutas y neurólogos), pero no para determinar potencia muscular en individuos normales.

La fuerza generada por el aductor del pulgar en respuesta a una estimulación eléctrica de] nervio cubital es una medida adecuada para determinar el estado nutricional. En individuos severamente desnutridos y después recuperados, se ha observado que los pacientes desnutridos generan menos fuerza, se fatigan más fácilmente y se relajan más lentamente; pero una vez. realimentados vuclven rápidamente a la normalidad, si bien el peso y otros indicadores bioquímicos de desnutridos permanecen bajo to normal ${ }^{10}$. Esto sugierc que la función muscular es un buen indicador del estado nutricional ${ }^{2-6}$.

Nuestros resultados son similares a los descritos anteriormente. Los sujetos con antecedentes de haber sufrido desnutrición, aun cuando de menor talla y peso que los controles, no mostraron dilerencias significativas en la composición corporal ni la nutrición de hierro con estos últimos, sin embargo, la contracción del aductor del pulgar fue menos potente y la recuperación muscular más lenta. No obstante, no se encontró correlación entre función muscular y estado nutricional medido por la composición corporal.

Los datos aportados por nuestros resultados sugieren que la contracción del músculo aductor del pulgar que sigue a la estimulación eléctrica del nervio cubital es un buen indicio del estado nutricional pasado, que podría relacionarse con la presencia de reservas de energía ${ }^{19}$. El mayor aporte de energia para la contracción muscular proviene de la adenosina trifosfato (ATP). En las contracciones breves, de menos de $10 \mathrm{~s}$ de duración $\mathrm{y}$ en las contracciones isométricas de $70 \%$ de la fuerza máxima, la energía necesaria para la contracción se obtiene de reservas locales, en cambio en las actividades que duran algo más tiempo se debe regenerar ATP por refosforilación de fosfocreatina y a partir de la glicolisis anaeróbica que produce aumento de lactato. La fuerza de contracción en preparaciones de músculo aislado depende del abastecimiento de ATP por fosfocreatina. En contraste con el músculo en actividad sostenida, que requiere de la glicolisis aeróbica para obtener su energía por fosforilación oxidativa, el músculo en reposo no requiere de la fragmentación del glicógeno para proporcionarse energía y $60 \%$ de la requerida para su mantención en estas condiciones puede ser obtenida directamente de hidratos de carbono que difunden a los tejidos desde la sangre.

La prevalencia de pacientes hospitalizados con deterioro nutricional es cada día más importante y se ha señalado que llegaría hasta $50 \%$. Con el aumento de tratamientos ambulatorios a enfermos crónicos, el conjunto de pacientes que requieren hospitalización deberá incluir mayor proporción de personas con más deficiencias nutricionales, por lo que parecería recomendable determinar, al ingreso de cada uno de ellos, el estado nutricional por métodos de antropometría y otros, incluyendo la función muscular.

\section{Resumen}

La función muscular se relaciona con estado nutricional. Se estudió función muscular esquelética en 40 niños con antecedentes de haber sufrido una desnutrición grave precoz (GE) en comparación con igual número de niños pareados por sexo y edad a los 12 a $4 \mathrm{~m}$, sin antecedentes de desnutrición (GC). La función muscu- 
lar se midj6, según el protocolo de Shizgal, de la fuerza de contracción isométrica del músculo abductor del pulgar obtenida por estimulación eléctrica del nervio cubital (en la muñeca) y registrada mediante un transductor de fuerza (Grass Mod FT-10). La fuerza muscular se relacionó con la composición corporal determinada por antropometría convencional y las fórmulas de Durnin y Frisancho. La recuperación a la fatiga muscular fue más rápida $(p<0,05)$ y la fuerza generada mayor $(p<0,05$ ) en los niños controles, sin antecedente de desnutrición. La desnutrición grave precoz altera la función muscular, incluso pasados varios años después de su tratamiento y recuperación.

(Palabras clave: trastornos de la nutrición. desnutrición, contracción muscular.)

\section{Agradecimientos}

Al Dr. Alejandro Herrández K., por la revisión y comentarios del manuscrito, como también a las Sras. Margarita Vial y Catmen Artaza por su cooperación a este estudio.

\section{Referencias}

1. Krishamurtity $N$, Balakumar B. Thombre DP: Effects of undernutrition and sex difference on skeletal muscle function in young rats Indian. J Physiol Pharmacol 1992: 36 234-238.

2. Shizgol HM, et al.: Nutritional assessment and skeleta] muscle function. Am J Clin Nutr 1986; 44: 76I-771.

3. Reeds PJ, Jackson AA, Pic'ou D. Pulter N: Muscle mass and composition in malnourished infants and children and changes seen after recovery. Pediatr Res 1978: 12:613-618.

4. Hatsen-Smith FM, Picou D. Golden MH: Growth of muscle fibres duting rccovery from severe malnutrition in Jamaican infants. Br J Nutr 1979; 41: 275 282.

5. Cheek DB. Hill DE. Cordano A. Graham GG: Malnutrition in infoncy: changes in muscle and adipose tissue before and after rehabilization. Pediatt $\operatorname{Res} 1970 ; 4: 135-144$.
6. Forbes GB, Bruining GJ: Uninary creatinine excretion ard lean body mass. Am J Clin Nutr 1976: 29: 13591360 .

7. Waleriow $J C$, Mendes $C B$ : Composition of muscle in malnourished human infants. Nature 1957: 180: 13611362.

8. Mac Lean WC. Grairam GG: The effect of energy intake on nitrogen content of weight gained by tecovering malnourished infants. Am J Clin Nutr 1980 : 33: $903-909$

9. Hosking GP. Bhat US, Dabowitz V, Edwards RHT. Measurements of muscle strength and perfomance in children with nomal and diseased muscle. Arch Dis Child 1976: 51:957-963.

10. Rursell DM, Leiter LA, Whitwell I, Marliss EB. Jeejeebboy $K N$ : Skeletal muscle function during hypocaloric diets and fasting: a comparison with standard nutritional assessment parameters. Am J Clin Nutr 1983: 37: 133-138

IJ. Moxhan I. Morris AJR. Spiro SG, Edwards RHT. Green $M$ : Contractile properties and fatigue of the djap̀ragm in man. Thorax 1981: 36: 164-168.

12. Alvear J. Artaza C. Vial M. Guerrero S. Muzzo $S$. Physical growth and bone age of survivors of protein energy malnutrition. Asch Dis Child 1986; 61: 257. 262 .

13. Alvear $f$, Vial $M$, Artaza C: Crecimiento despues de desnutrición grave precoz. Rev Chil Pediatr 1991; 62: $242-247$.

14. Smith $D$ : Measurements, methods and slandards. In: Smith D, ed. Growth and its disorders: Basic and standard approach and clasification. growth deficiency disorders. growth excess disorders, obesity. Phyladelphia: W.B. Saunders Compary 1977: 18-61.

15. Frisancho A: New norms of upper limb fat and muscle areas for assessment of nutritional status. Am J Clin Nutr 1981: $34: 2540-2545$.

16. Sann L. Durand M, Picard J. Lasne Y. Behtennd M: Arm fat and muscle areas in infancy. Arch Dis Child 1988; 63: 256-360.

17. US Departament of Health Education and Welfare Public Health Service NCHS. Curves for children: Birth to 18 years. United States DHEW 1977. Vital and Health Statistics. Serie 11 , Number 165, 1977.

18. Simes MA, Addiego JE Jr, Dallman PR: Ferritin in serum; diagnosis of iron deficiency and iron overload in infants and children. Blood 1974: 43: 581-590.

19. Edwards R. Young A, Hosking GP, Jones DA: Human skeletal muscle function: Description of test and normal value. Clin Sci Molec Med 1977: 52: 283-290.

20. Forse RA. Shizgal HM; The assessment of malnutrition. Surgery 1980; 88: 17-24. 